\title{
Facilitating the adaptability of buildings through the separation of components
}

\author{
Shabtai Isaac $^{1 *}$, and Farnaz Sadeghpour ${ }^{2}$ \\ ${ }^{1}$ Department of Structural Engineering, Ben-Gurion University of the Negev, Israel \\ ${ }^{2}$ Department of Civil Engineering, University of Calgary, Canada \\ *Corresponding author (isaacsh@bgu.ac.il)
}

\begin{abstract}
Purpose Buildings need to be adaptable with relative ease to new user requirements, regulations or technologies. Adaptability reduces the effort and expense involved in adding, changing or replacing building components (such as partitions, doors or plumbing fixtures), throughout the building's life-cycle. This increases the buildings' value and sustainability, as well as the building user's satisfaction. In practice, however, most buildings are designed and constructed to suit their current use, while their future adaptability is ignored. Our research follows an approach that is based on the systematic separation of building components whose replacement occurs at different intervals. Such a separation reduces the efforts, waste and costs currently involved in adapting buildings to the changing needs of their users. Method A number of methods are used in order to support the design of adaptable buildings. The building components in the design are ordered through pair-wise comparisons of their replacement rates. This is preferable to an assessment of the actual life expectancies of the components' in light of the uncertainty regarding external factors such as maintenance policies and future technologies. The relationships between specific building components with different replacement rates are then detected using graph-based methods. A clustering algorithm is applied to a weighted graph representing the design, in order to distinguish between groups of components with different replacement rates. Building components with different replacement rates are then separated through the systematic application of buffers in the building design. A scenario-based method is used to evaluate the costs and benefits of these buffers. A path-search algorithm 5 automatically identifies the components that will be affected by changes in each scenario. Results \& Discussion Preliminary research included an implementation of the proposed methods in a small-scale case study. Results of this test gave an indication of the feasibility of these methods, suggesting that they might support the design of adaptable buildings. This could facilitate an alternative to current housing policies for elderly people - i.e. adjusting buildings according to the frequently changing needs of their inhabitants, instead of requiring the inhabitants to keep moving as they need more assistance.
\end{abstract}

Keywords: Management \& Social issues, Design Management, Adaptable Buildings, Graph-based Methodology

\section{INTRODUCTION AND RESEARCH OBJECTIVES}

Most buildings are constructed at a significant cost and are expected to be functional for many decades to come. For that to happen, buildings have to be adaptable: it should be possible to adjust them with relative ease to the changing needs of their users and to any new regulations or technologies that are introduced subsequent to their construction ${ }^{8}$. Housing for elderly people in particular needs to respond to frequent changes in the users' needs ${ }^{7,22}$. Adaptability reduces the effort and expense involved in adding, changing or replacing the components of a building throughout its life-cycle. A building component is a product that forms a distinct unit, and has its own functional identity, such as window, a partition wall or a beam. A component may contain a number of different materials, but it is usually constructed or assembled in a single process.
Increasing the adaptability of buildings is one of the most effective ways to increase their value, and their users' satisfaction ${ }^{20}$. Non-adaptable buildings are also less sustainable, since they require the demolition and replacement of many components in order to accommodate changes - components that will usually end up in an incinerator or landfill.

In practice, most buildings are, however, designed and constructed to suit their use at that time, and their future adaptability is ignored ${ }^{3}$. Often, the only way to eventually accommodate changes is through extensive and expensive refurbishments. Elderly people, for example, often have to keep moving to new homes as they need more assistance, because their houses cannot be easily adapted to their changing needs.

The objective of this research is to develop a methodology that supports the design of buildings 
that can be gradually adapted throughout their use. The methodology is based on the systematic separation of building components whose replacement occurs at different intervals. These components are currently often connected to each other. Connections between components may be physical, such as the connection between one component that is supported or covered by another component, or they may be functional, such as the connection between two components that satisfy the same requirement (Figure 1).

For example, piping with a short physical life may be covered or hidden by tiling with a longer physical life. This may cause waste when the replacement of the piping requires the replacement of the tiling as well. Connections between building components with different replacement rates may cause the owner of a building to refrain from making changes altogether. For example, changes to a heating, ventilation and air-conditioning (HVAC) system may require expensive changes to the structure, because ceiling heights limit the space available for ducts. The separation of frequently replaced piping from the tiling that covers it, or of frequently replaced ducts from the ceiling above them, can facilitate the building's adaptability.

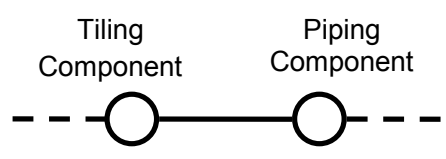

a. Direct physical connection

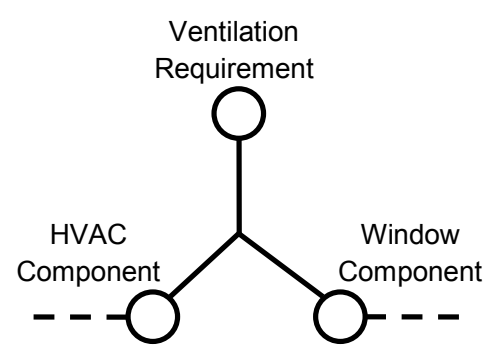

b. Indirect functional connection

\section{Fig. 1. Connections between components}

The separation of building parts to allow adaptability has been studied in previous research. The Dutch architect John Habraken in particular proposed the Open Building system, in which the 'base-building' and its interior are separated ${ }^{10}$. Most of the previous research has focused on the separation of building systems ${ }^{11,19}$. A building system is an assembly of components that satisfies particular user requirements, such as the structure, cladding, mechanical systems, etc. Each system is usually designed and constructed by different specialists. Connections may exist between the components within a system, as well as between components that belong to different systems. The representation of the different systems in a single integrated model is a prerequisite for the development of a methodology that facilitates their separation.

Durmisevic and Brouwer ${ }^{6}$ have proposed to extend this approach in order to include the separation not only of entire building systems, but also of individual components within a system, which may have different replacement rates. The application of such an approach is particularly challenging in the design of large buildings, which contain thousands of different components that are interconnected through various types of relationships. On the other hand, this approach can be especially effective in such large buildings, when they are built and maintained throughout their lifecycle by the same owners (e.g. hospitals, office buildings, elderly housing, etc.).

In this research, graphs are used to represent the design of buildings. Graphs have been used to model complex systems that consist of many highly interconnected elements ${ }^{4}$. A graph-based representation of projects can facilitate a systematic analysis which takes into account a large number of components simultaneously. Moreover, it allows the use of different graphtheoretic tools and algorithms to partially automate such an analysis - an essential feature when the complexity of the system crosses a certain threshold $^{15}$.

The separation of components can be seen as a form of modularization, i.e., the decomposition of the building into modules. These modules are products that may contain a number of components, and that have carefully designed interfaces with other modules. Modular construction currently focuses on the use of prefabricated three-dimensional modules and twodimensional panels with certain standard dimensions and interfaces which are repeated throughout the building ${ }^{2}$. The definition of these dimensions and interfaces is based on the requirements of the production, transportation and installation processes of the modules. 
The present research, on the other hand, also addresses the functionality of the modules, allowing them to have different dimensions, but not components with different replacement rates. This may facilitate not only the efficient preassembly of the module, but also its efficient replacement. The functionality of the modules is addressed through an analysis of the requirements satisfied by the components in the module. The definition of the requirements in the project brief may thus have an impact on the definition of modules in the design. For example, the requirements in one project may justify the design of a façade panel as a single module which can be easily replaced, while in another project they may justify the design of the panel as an assembly of a number of modules, each of which can be separately replaced.

Attempts to achieve adaptability have, until now, focused on the design of component interfaces that are:

- Flexible, allowing components to be easily moved, such as a facade with sliding shutters that can be changed by the user, or reconfigurable sliding walls ${ }^{18}$.

- Standardized, allowing one type of component to be easily replaced with another type in a modular system such as a façade or partitioning system ${ }^{9}$.

Buffers in the design can also be used to separate components and contribute to the long-term adaptability of buildings. Buffers can be created through the design of components with a larger capacity. For example, larger service-cores can allow additional pipes and ducts to be installed in the future, without requiring changes to the structure of the service-cores. Other buffers may consist of a larger number of components. For example additional vertical waste pipes can allow new sanitary appliances to be connected in the future without requiring changes to the drainage system.

\section{SUPPORTING THE DESIGN OF ADAPTABLE BUILDINGS}

The objective of this research is to develop a methodology that supports the design of adaptable buildings through a systematic separation of building components whose replacement rates differ significantly. The methodology can be used for the detailed design of buildings, once the project brief and the initial design have been defined. It consists of a number of processes (Figure 2):

a. Representation of the building design and project brief as a graph

b. Ordering the building systems according to their relative replacement rates

c. Adjustment of the replacement rates of building components according to requirements in the project brief

d. Separation of components with different replacement rates through the use of buffers and flexible interfaces in the building design

The following sections explain each process in the methodology in more detail:

\section{Representation of the design and brief as a graph}

In the graph-based representation of the design that was developed in this research, building components are represented as nodes, and the connections between components as links between the nodes. The graph can be created automatically by extracting information from existing object-oriented models of construction projects, such as Building Information Models $(\mathrm{BIM})^{21}$. These models contain objects which represent the building components belonging to different systems in the design, as well as information on the physical connections between components.

In order to represent functional connections between components in the graph, the user requirements in the project brief are also represented as nodes. A novel method is used to connect the requirements to building components in the project design, creating one integrated project representation.

The nodes in the graph representing the requirements and components are connected through a third type of nodes called design subjects. The design subject nodes have attributes defining the components that satisfy a specific project requirement ${ }^{13}$. For example, a "lighting" design subject links a requirement for a certain level of illumination in a space, with the window and lighting fixture components in that same space (Figure 3). 


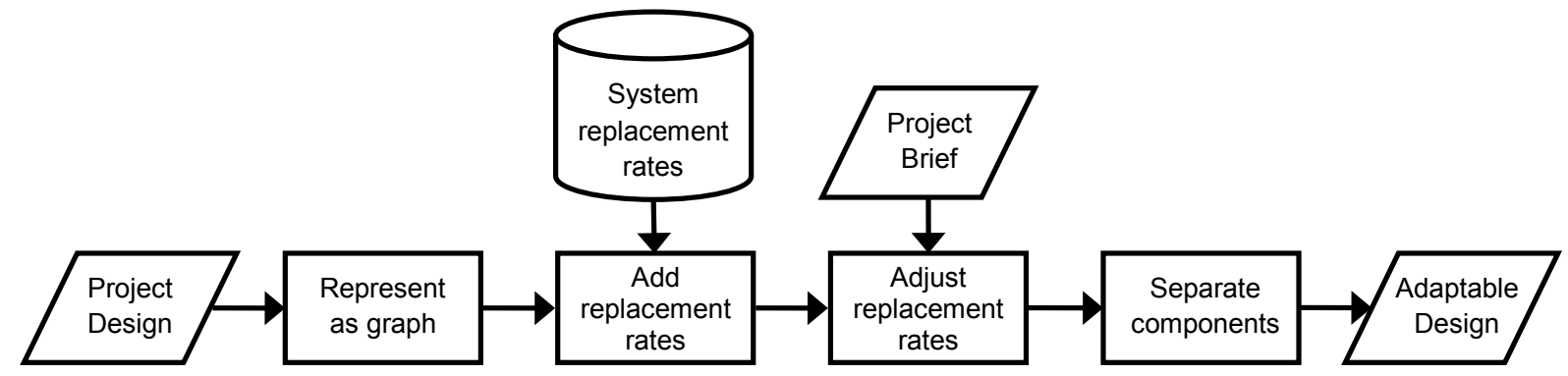

Fig. 2. A methodology supporting the design of adaptable buildings

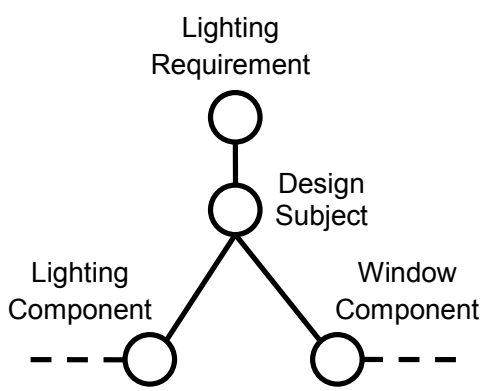

Fig. 3. Linking requirements and components in the graph-based model

Graph Transformation (GT) rules can be applied to automatically connect the components and requirements through the design subjects ${ }^{15}$. GT is a technique for automatically implementing changes in graphs through predefined rules ${ }^{12}$. The GT rules specify how the graph should be built, and how it can evolve. Using GT rules, the model can also be updated according to changes which are proposed for the components in the design.

\section{Ordering the building systems according to their replacement rates}

In the second stage, building systems in the project's design, or parts of these systems, are ordered according to an initial assessment of their replacement rates. These replacement rates are stored in a generic database, and are based on pair-wise comparisons by experts, rather than on an assessment of the actual size of the life expectancies of the systems.

Replacement rates of building components have been assessed in life-cycle research. However, despite being the subject of numerous studies, lifecycle research has not had much of an impact on practitioners and decision-makers in the construction industry ${ }^{5,16}$. An important reason for this is that such research has focused mostly on the physical obsolescence of building components, while their actual replacement rate is more often determined by external factors, such as maintenance policies, changes in the use of a building, new regulations, or the introduction of new technologies ${ }^{17}$. These factors are difficult to predict, and there is consequently a general lack of knowledge regarding the actual life expectancy of building components ${ }^{1}$.

It may be clear, for example, that the building structure is likely to last longer than engineering services components, and that some of these components will last longer than finishes and fittings, but it can be very difficult to determine the exact number of years they will last. It is therefore more feasible to use an order topology for such an assessment, instead of a metric topology. In other words, it is possible to assess which system is likely to be replaced sooner, even when it is difficult to assess when exactly this will happen.

The assessed relative replacement rates are defined as attributes of the building components, according to the systems to which they belong (Figure 4). Components are often connected in the design to other components that belong to different systems, and which therefore have different replacement rates. The representation of the components as nodes in a graph, with attributes defining their relative replacement rates, allows an identification of the similarity or difference between the replacement rates of any two connected components. 


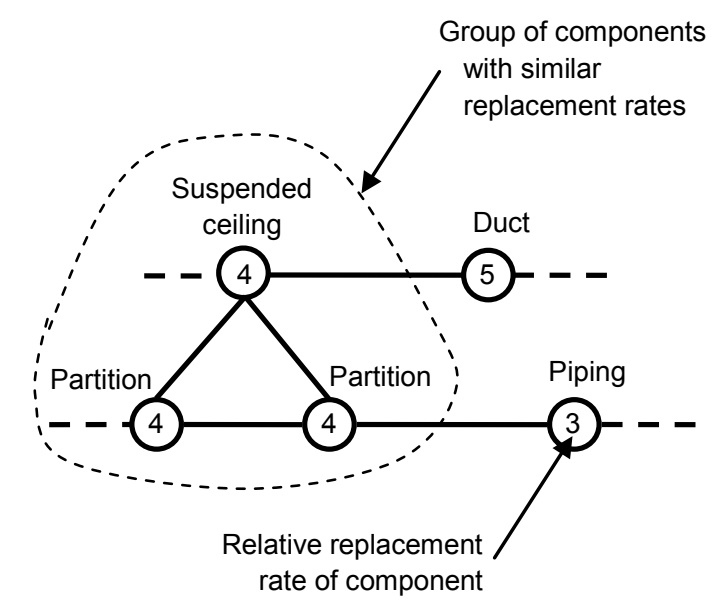

Fig. 4. Defining the initial relative replacement rates of the components

\section{Adjustment of replacement rates of the components}

The initial assessment of the replacement rates of the components is based on their expected physical obsolescence rates, and on typical maintenance policies and changes in use. However, the functional requirements in a specific project may cause the replacement rates to be different. For example, the expected tenant turnover in a project may have a significant impact on the replacement rate of certain components. The implications of different scenarios for future changes to the requirements are therefore identified and analyzed by the project team. The components that satisfy a requirement that could change in the future, and which will therefore be affected by this change, can be identified in the graph. This can be done automatically using a path search algorithm that traces the links that connect the project requirements to the components ${ }^{14}$. The path search algorithm identifies the shortest path between the node in the graph representing the requirement that will change, and the nodes representing the components that will be affected by this change.

The replacement rates of the affected components are adjusted when changes are expected to occur sooner than the assessed replacement rate of the building system to which they belong. For example, if a future change in a requirement will lead to the replacement of a component before it is physically obsolete, its replacement rate is adjusted accordingly. The adjusted replacement rates often do not conform to the preconceived decomposition of the project into systems. Thus, components which belong to different systems, but are affected by the same change, will have the same replacement rates.

\section{Separation of components with different replacement rates}

Following the adjustment of the replacement rates of the components, groups of components with similar replacement rates can be identified. These groups are represented by clusters of nodes in the graph that have the same attributes. The identification of the clusters of nodes in a graph can be automated through the use of graph clustering algorithms ${ }^{14}$. Connections between components in different groups can thus be identified. The existence of such connections indicates that changes to components with high replacement rates may require otherwise unnecessary changes to components with lower replacement rates. The objective in this stage is therefore to systematically reduce the dependencies between components with different replacement rates, which are represented by the links between clusters of nodes in the graph. This will allow the building to be more easily adapted at some point in the future.

Groups of building components with different replacement rates are separated through a systematic provision of buffers in the building design, and through the design of modules with standard and flexible interfaces:

a. Buffers are applied to the attributes of components, such as size or number, to reduce the dependency between components and absorb the impact of changes. For example, certain components in the building structure can be designed to withstand larger loads than currently required, in order to be able to accommodate future changes or additions of other building components.

b. Standard interfaces are designed to enable a flexible connection between modules which contain components with different replacement rates. For example, a separation of piping from walls through flexible connections can allow the replacement of pipes without damaging the wall.

Naturally, the choice between introducing either buffers or flexible connections in the design has to be based on an economic evaluation of the cost of each solution.

\section{ILLUSTRATIVE CASE}

In order to investigate the feasibility of the proposed methodology, a simple illustrative case of a one-bedroom apartment for an elderly couple, which contains one bathroom, is used. Building 
systems in the apartment are ordered according to their assessed replacement rates:

$\begin{aligned} \text { i. } & \text { Structure } \\ \text { ii. } & \text { Exterior walls } \\ \text { iii. } & \text { Windows and exterior cladding } \\ \text { iv. } & \text { Interior partitioning } \\ \text { v. } & \text { Plumbing }\end{aligned}$

The replacement rates of the building components in the design are defined according to the systems to which they belong (e.g. "1" for components belonging to the building structure). The design of the apartment is represented as a graph, in which

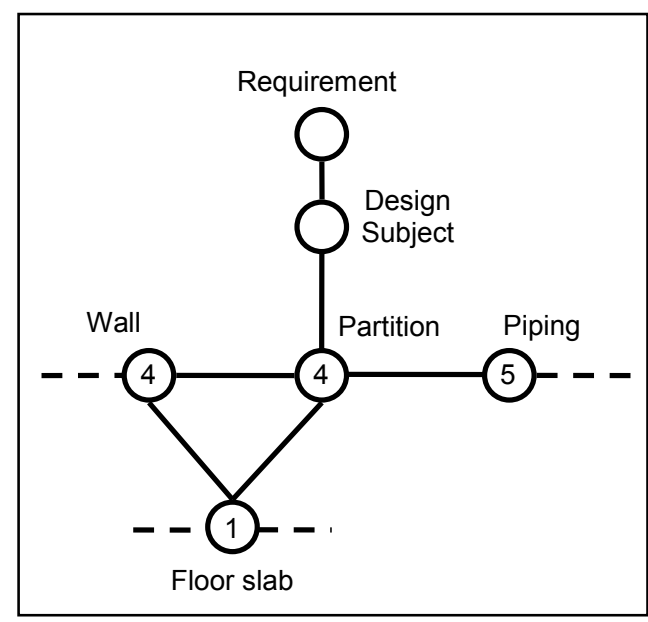

a. Initial definition of replacement rates each building component is represented as a node. Figure 5 shows four of the components, with their replacement rates.

In the next stage, these replacement rates are adjusted by identifying user requirements in the project brief that will likely change in the future. In this case, possible changes in the requirements for the bathroom are identified. Ensuring access and safety for the users as they grow older may require adaptations to the bathroom. Two changes to the requirements are examined:

a. A bathtub is replaced with a shower.

b. The accessibility of the bathroom is improved.

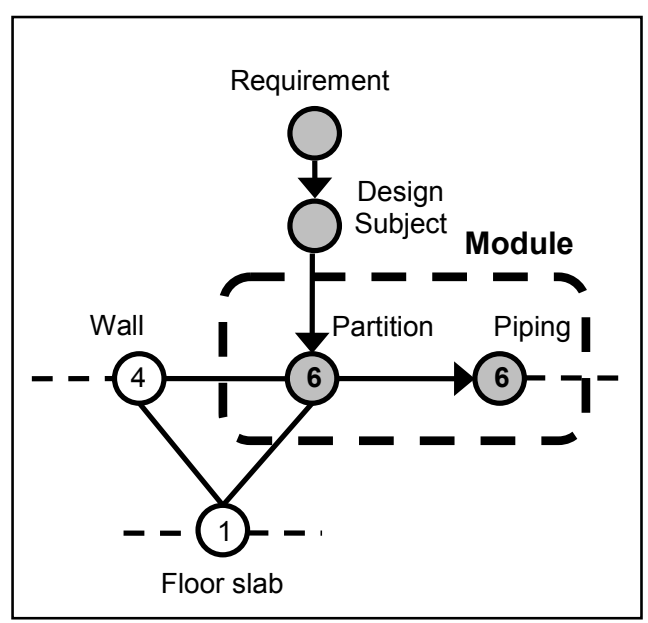

b. Adjustment of replacement rates

Fig. 5. Adjusting the replacement rates of components

The components that will be affected by these changes are identified by tracing links in the graphbased model that connect the changed requirements to the affected components. Other components, which are physically connected to the components directly affected by the changes, can also be identified by tracing links between the nodes in the graph. Thus, replacing the bath with a shower will also require changing the piping in a wall inside the bathroom, as well as changing the type of window next to the shower. Making the bathroom accessible will require moving a partition wall with the piping inside it, as well as a window next to that wall.

The relative replacement rates of the affected components are adjusted by examining whether the changes in the requirements are expected to occur sooner than the previously assessed replacement rates of the building systems. In this case, the changes are expected to occur sooner than the date at which the plumbing would otherwise have to be replaced. In other words, all the affected components will be changed sooner than their usual replacement rates require. The replacement rate attribute of these components is therefore changed to "6" (Figure 5).

Once the replacement rates have been adjusted, the nodes in the graph are grouped accordingly. Critical links between groups of components with different replacement rates are then identified. These include, for example, links between a window or a pipe that will have to be changed, and other adjacent components whose replacement is not required. The critical links are adjusted to reduce such dependencies, through the design of the interfaces of the components, and through the use of buffers:

a. In the case in which the bathtub is replaced by a shower, the interfaces of components can be designed to allow the expected changes. Thus, the partition wall can be designed to have a demountable covering, so that the piping that 
runs through it can be changed without damaging the tiling on the wall. The window can be designed so that it can be changed without removing the entire window unit, keeping the frame and the tiling around it intact. In other words, both partition wall and window are not treated as a single module, but rather as a set of components with different replacement rates.

b. In the case of the change in which accessibility of the bathroom is improved, other solutions can be applied. One solution is to design a partition wall as a single module that can be easily demounted and moved, together with the tiling on the wall and the piping within it (Figure 5b). Similarly, the window and adjacent sections of the exterior wall can be designed as modules with interfaces that allow them to be easily changed, without it affecting the building structure. An alternative solution is based on the use of a buffer: an increase in the current size of the bathroom. The choice between installing modular walls and increasing the size of the bathroom has to be based on an economic evaluation of the cost of each solution. Naturally, additional solutions can be proposed which contain both buffers and modules with standard interfaces.

\section{Conclusions}

This paper presents a methodology that supports the design of adaptable buildings through a separation of building components whose replacement rates differ. The methodology contains a number of processes which allow the identification of the relative replacement rates of building components, and the separation of components with different rates. It makes use of graphs to represent the design of buildings, and of graph-theoretic tools to facilitate a systematic analysis of complex buildings that contain a large number of components. The separation of components is achieved through the provision of buffers in the design, and through the design of modules with standard and flexible interfaces.

Modules are defined by their functionality, in addition to their physical dimensions, through an analysis of the user requirements satisfied by their components. Likely future changes in these requirements can be translated into a different modularization of the building. For example, in the illustrative case described in this paper, a partition wall was defined as either one module, or as a number of connected modules, depending on the expected changes. Future research will focus on the question how preassembled modules can be used to facilitate both their efficient installation in a new building, as well as an efficient adaptation of existing buildings. This is likely to be relevant in particular regarding preassembled modules containing mechanical, electrical, and plumbing components.

\section{REFERENCES}

1. Ashworth, A., "Assessing the life expectancies of buildings and their components in life cycle costing", COBRA 96, RICS, 1996.

2. Balaguer, C., Abderrahim, M., Navarro, J.M., Boudjabeur, S., Aromaa, P., Kahkonen, K., Slavenburg, S., Seward, D., Bock, T., Wing, R., Atkin, B., "FutureHome: An integrated construction automation approach", Robotics \& Automation Magazine, IEEE, Vol. 9(1), pp. 5566, 2002.

3. Beadle, K., Gibb, A., Austin, S., Fuster, A., Madden, P., "Adaptable Futures: Setting the Agenda", $1^{\text {st }}$ International Conference on Industrialised, Integrated, Intelligent Construction, Loughborough, UK, pp. 35-44, 2008.

4. Boccaletti, S., Latora, V., Moreno, Y., Chavez, M., Hwang, D.U., "Complex networks: Structure and dynamics", Physics Reports, Vol. 424(4-5), pp. 175-308, 2006.

5. Cole, R.J., Sterner, E., "Reconciling theory and practice of life-cycle costing", Building Research and Information, Vol. 28(5/6), pp. 368-375, 2000.

6. Durmusevic, E., Brouwer, J., "Design Aspects of Decomposable Building Structures", the CIB Task Group 39, Design for Deconstruction and Materials Reuse, Karlsruhe, Germany, pp. 81103, 2002.

7. Frain, J.P., Carr, P.H., "Is the Typical Modern House Designed for Future Adaptation for Disabled Older People?", Age and Ageing, Vol. 25(5), pp. 398-401, 1996.

8. Geraedts, R., "Future Value of Buildings", The $3^{\text {rd }}$ CIB Conference on Smart and Sustainable Environments, Delft, Netherlands, 2009.

9. Gibb, A., Austin, S., Dainty, A., Davison, N., Pasquire, C., "Towards Adaptable Buildings: pre-configuration and re-configuration - two case studies", in: M. Sharp (Ed.), ManuBuild 1st International Conference, Rotterdam, Netherlands, pp. 149-159, 2007.

10. Habraken, N.J., "Open Building as a condition for industrial construction", $20^{\text {th }}$ International Symposium on Automation and Robotics in Construction, Eindhoven, the Netherlands, 2003, pp. 37-42.

11. Hansen, G.K., Olsson, N.O.E., "Layered Project-Layered Process: Lean Thinking and Flexible Solutions", Architectural Engineering and Design Management, Vol. 7(2), pp. 70-84, 2011. 
12. Heckel, R., "Graph Transformation in a Nutshell", Electronic Notes in Theoretical Computer Science, Vol. 148, pp. 187-198, 2006.

13. Isaac, S., Navon, R., "Feasibility Study of an Automated Tool for Identifying the Implications of Changes in Construction Projects", Journal of Construction Engineering and Management Vol. 134(2), pp. 139-145, 2008.

14. Isaac, S., Navon, R., "A Graph-based Approach to the Modeling of Changes in Construction Projects", 28 ${ }^{\text {th }}$ International Symposium on Automation and Robotics in Construction, Seoul, Korea, pp. 66-71, 2011.

15. Isaac, S., Navon, R., "Modeling Construction Projects as a Basis for Change Control", Automation in Construction, Vol. 18(5), pp. 656-664, 2009.

16. Langston, C., "Life-Cost Approach to Building Evaluation", Butterworth-Heinemann, Oxford, 2005.

17. Langston, C., Wong, F.K.W., Hui, E.C.M., Shen, L., "Strategic assessment of building adaptive reuse opportunities in Hong Kong", Building and Environment, Vol. 43(10), pp. 1709-1718, 2008

18. Lelieveld, C.M.J.L., Voorbij, A.I.M., Poelman, W.A., "Adaptable architecture", in: Y. Kitsutaka (Ed.), Building stock activation, TAlHEI Printing Co., Tokyo, pp. 245-252, 2007.

19. Leupen, B., "Frame and Generic Space", 010 Publishers, Rotterdam, 2006.

20. Manewa, A., Pasquire, C., Gibb, A., Schmidt, R., "Towards Economic Sustainability through Aadaptable Buildings", The $3^{\text {rd }}$ CIB Conference on Smart and Sustainable Environments, Delft, Netherlands, 2009.

21. Nguyen, T-H., Oloufa, A.A., Nassar, K., "Algorithms for automated deduction of topological information", Automation in Construction, Vol. 14(1), pp. 59-70, 2005.

22. Struyk, R., Katsura, H., How the Elderly Adjust Their Housing Without Moving, Routledge, 1988. 